\title{
Close coupling test of classical and semiclassical cross sections for rotationally inelastic Ar-N2 collisions
}

Russell T. Pack

rt7mhpack@msn.com

Follow this and additional works at: https://scholarsarchive.byu.edu/facpub

Part of the Biochemistry Commons, and the Chemistry Commons

\section{Original Publication Citation}

Pack, Russell T."Close coupling test of classical and semiclassical cross sections for rotationally inelastic Ar-N[sub 2] collisions." The Journal of Chemical Physics 62 (1975): 3143-3148.

\section{BYU ScholarsArchive Citation}

Pack, Russell T., "Close coupling test of classical and semiclassical cross sections for rotationally inelastic Ar-N2 collisions" (1975). Faculty Publications. 782.

https://scholarsarchive.byu.edu/facpub/782 


\title{
Close coupling test of classical and semiclassical cross sections for rotationally inelastic $\mathrm{Ar}-\mathbf{N}_{\mathbf{2}}$ collisions ${ }^{*}$
}

\author{
Russell T Pack \\ Department of Chemistry, Brigham Young University, Provo, Utah 84602 \\ (Received 25 November 1974)

\begin{abstract}
Accurate quantum mechanical close coupling (CC) integral cross sections are reported for rotationally inelastic $\mathrm{Ar}-\mathrm{N}_{2}$ collisions at room temperature using an empirical intermolecular potential. These cross sections are used to test the infinite order sudden (IOS) approximation and the results of several methods [classical trajectories (CT), full sudden (SA), generalized phase shift (GPS), and classical limit generalized phase shift (CGPS)] as reported in the preceding paper by Pattengill. The IOS approximation works very well for cross sections involving low rotational states. The CT method works well for all the cross sections examined, justifying the popular belief that the rotational motion of $\mathbf{N}_{2}$ is essentially classical at room temperature.
\end{abstract}

\section{INTRODUCTION}

In the preceding paper, Pattengill ${ }^{1}$ compares the results of several semiclassical approximations with exact classical calculations of rotationally inelastic integral cross sections for $\mathrm{Ar}-\mathrm{N}_{2}$ collisions. In the present paper, we report accurate quantum mechanical close coupling (CC) calculations on the same system as a test of both the classical and semiclassical results. In addition, we also report results for one additional simple approximation, the infinite order sudden (IOS) approximation. ${ }^{2-4}$

The $\mathrm{Ar}-\mathrm{N}_{2}$ intermolecular potential used is an empirical $12-6$ potential with $P_{2}(\cos \theta)$ anisotropies. Its parameters were first specified by Pattengill, La Budde, Bernstein, and Curtiss ${ }^{5}$ and have since been listed several times, ${ }^{1,3,6}$ so that they need not be repeated here. This intermolecular potential is probably a reasonably accurate one for this system; however, recent a priori calculations of intermolecular potentials for $\mathrm{Ar}-\mathrm{CO}$ and other atom-molecule systems ${ }^{7}$ indicate that the present repulsive anisotropy parameter is probably too small, and that a $P_{4}(\cos \theta)$ term is also needed to describe the repulsive potential. Hence, the present intermolecular potential should be viewed as a realistic model but comparison with experiment not taken too seriously.

The CC calculations reported herein were done using Gordon's ${ }^{8}$ method and program with only minor modifi- cations.

The IOS approximation, which has recently been generalized to arbitrary intermolecular potentials by Hunt$\mathrm{er}^{9}$ and by Secrest, ${ }^{10}$ has been discussed in several previous publications, ${ }^{2-4}$ but it may be well here to point out its relationship to the sudden approximation (SA) used by Pattengill in the preceding paper. ${ }^{1}$ The IOS approximation used here is formulated in a body-fixed coordinate system ${ }^{4}$ and consists of two approximations:

(1) The neglect of coupling between different values of the body-fixed $z$ component of the angular momentum. $\mathrm{We}^{4}$ have called this the "centrifugal sudden" approximation, and McGuire and Kouri" ${ }^{11}$ have called it the " $J_{z}$ conserving" approximation. If formulated in the usual space-fixed coordinates it is equivalent to neglecting differences between the centrifugal potentials appearing in the coupled radial Schrödinger equations. ${ }^{4}$ (2) The neglect of the differences in energies of the rotational states that are coupled together. This is the "energy sudden" approximation. Except for the use of WKB phase shifts no other approximations are made. The resulting IOS scattering matrix (for a given total angular momentum $J$ ) has the exactly unitary form

$$
\mathbf{S}=\exp \left(2 i U \eta U^{\dagger}\right)
$$

where $U$ is a unitary transformation matrix and $\eta$ is a

TABLE I. Accurate CC integral cross sections $\sigma\left(j^{\prime}-j\right)$ for rotationally elastic and inelastic collisions of $\mathrm{N}_{2}$ with $\mathrm{Ar}$ with $E_{10} / k_{B}=300^{\circ} \mathrm{K}$ using an empirical intermolecular potential. The units are $\AA^{2}$. Most of the cross sections are believed accurate to within $4 \%$. Those less accurate are placed in parentheses or omitted. The values of $j$ shown specify the basis of rotational states used. [The last line gives the relative translational energy (in ${ }^{\circ} \mathrm{K}$ with $k_{B}$ being Boltzmann's constant) of the collision if the $\mathrm{N}_{2}$ molecule is in rotational state $j$.]

\begin{tabular}{|c|c|c|c|c|c|c|c|}
\hline$j 入 j$ & 0 & 2 & 4 & 6 & 8 & 10 & 12 \\
\hline 0 & $\cdots$ & $(3.3)$ & 1.64 & 1.10 & 0.40 & 0.057 & $(0.0022)$ \\
\hline 2 & $(16.0)$ & $\cdots$ & $\cdots$ & 5.3 & 2.50 & 0.52 & $(0.0265)$ \\
\hline 4 & 13.4 & $\cdots$ & (345) & 10.5 & 5.7 & 1.90 & $(0.141)$ \\
\hline 6 & 11.4 & 11.4 & 13.4 & 343 & 10.9 & 5.0 & $(0.55)$ \\
\hline 8 & 4.5 & 5.8 & 7.9 & 11.7 & 365 & 11.7 & (1. 91) \\
\hline 10 & 0.58 & 1.08 & 2.37 & 4.9 & 10.6 & 435 & $(7.7)$ \\
\hline 12 & $(0.015)$ & $(0.037)$ & $(0.117)$ & $(0.36)$ & $(1.14)$ & (5. 1) & $(460)$ \\
\hline$E_{f} / k_{B}\left({ }^{\circ} \mathrm{K}\right)$ & 618 & 601 & 560 & 497 & 410 & 300 & 167 \\
\hline
\end{tabular}


TABLE II. Accurate CC cross sections $\sigma\left(j^{\prime}-j\right)$ for $A r-\mathrm{N}_{2}$ collisions with $E_{10} / k_{B}=450^{\circ} \mathrm{K}$. The notation is that of Table I.

\begin{tabular}{cccccccc}
\hline \hline$j$ & & & & & & \\
\hline$j$ & 0 & 2 & 4 & 6 & 8 & 10 & 12 \\
\hline 0 & $\cdots$ & $(3.2)$ & 1.37 & 0.99 & 0.46 & 0.094 & $(0.0054)$ \\
2 & $(15.7)$ & $\ldots$ & 8.4 & 4.7 & 2.59 & 0.74 & $(0.056)$ \\
4 & 11.4 & 14.4 & $(316)$ & 9.7 & 5.3 & 2.35 & $(0.258)$ \\
6 & 10.8 & 10.5 & 12.8 & 341 & 9.7 & 5.2 & $(0.86)$ \\
8 & 5.7 & 6.6 & 7.9 & 11.0 & 334 & 10.9 & $(2.50)$ \\
10 & 1.15 & 1.86 & 3.5 & 5.9 & 10.8 & 359 & $(8.1)$ \\
12 & $(0.055)$ & $(0.119)$ & $(0.32)$ & $(0.81)$ & $(2.08)$ & $(6.8)$ & $(433)$ \\
$E_{j} / k_{B}\left({ }^{\circ} \mathrm{K}\right)$ & 768 & 751 & 710 & 647 & 560 & 450 & 317 \\
\hline
\end{tabular}

diagonal matrix of-phase shifts. These phase shifts are "infinite order" in the anisotropic part of the potential; i. e. , if $\eta_{i}$ is expanded in powers of the potential anisotropy, an infinite series results. If only the zeroth and first order terms are retained then, as we have shown in detail elsewhere, ${ }^{12}$ the approximation reduces to what we have called" the "first-order sudden" (FOS) approximation which, except for numerical details, is the same as the "full sudden approximation" (SA) used by Pattengill in the preceding paper. ${ }^{1}$ We hasten to point out that while the FOS or SA phase shifts are first order in the potential anisotropy, the $\mathbf{S}$ matrix is still an exactly unitary exponential if no further approximations are made. (See Pattengill's discussion of the deviations from unitary noted by him.) If one were to expand the SA $S$ matrix in powers of the phase shifts and keep terms only through first order, the result, which has sometimes been called the first-order sudden approximation, would be the sudden limit of the ordinary distorted wave approximation which is a very bad approxi- mation for the rotationally inelastic collisions considered herein.

\section{CALCULATIONS AND RESULTS}

Three sets of CC calculations were done. In the first (see Table I), the energy was chosen so that the relative kinetic energy with the $\mathrm{N}_{2}$ in rotational state $j=10$ corresponds to $300^{\circ} \mathrm{K}$ (i.e., $E_{10} / k_{B}=300^{\circ} \mathrm{K}$, where $k_{B}$ is Boltzmann's constant). Rotational states $j=0,2,4, \ldots$, 12 were included. This required solving 49 coupled equations of one parity and 42 equations of the other parity at each total angular momentum $J$, and the calculations were carried out at $J=0,10,20, \ldots 180$. In the second, (see Tables II and IV), the kinetic energy in the $j=10$ state corresponded to $450^{\circ} \mathrm{K}$, states $j=0$, $2, \ldots 12$ were included, so that 49 and 42 coupled equations were involved, and the calculations were carried out at $J=0,10,20, \ldots 200$. In the third set (see Tables III and IV) $E_{10} / k_{B}=450^{\circ} \mathrm{K}$ again, but the states $j=8,10$,

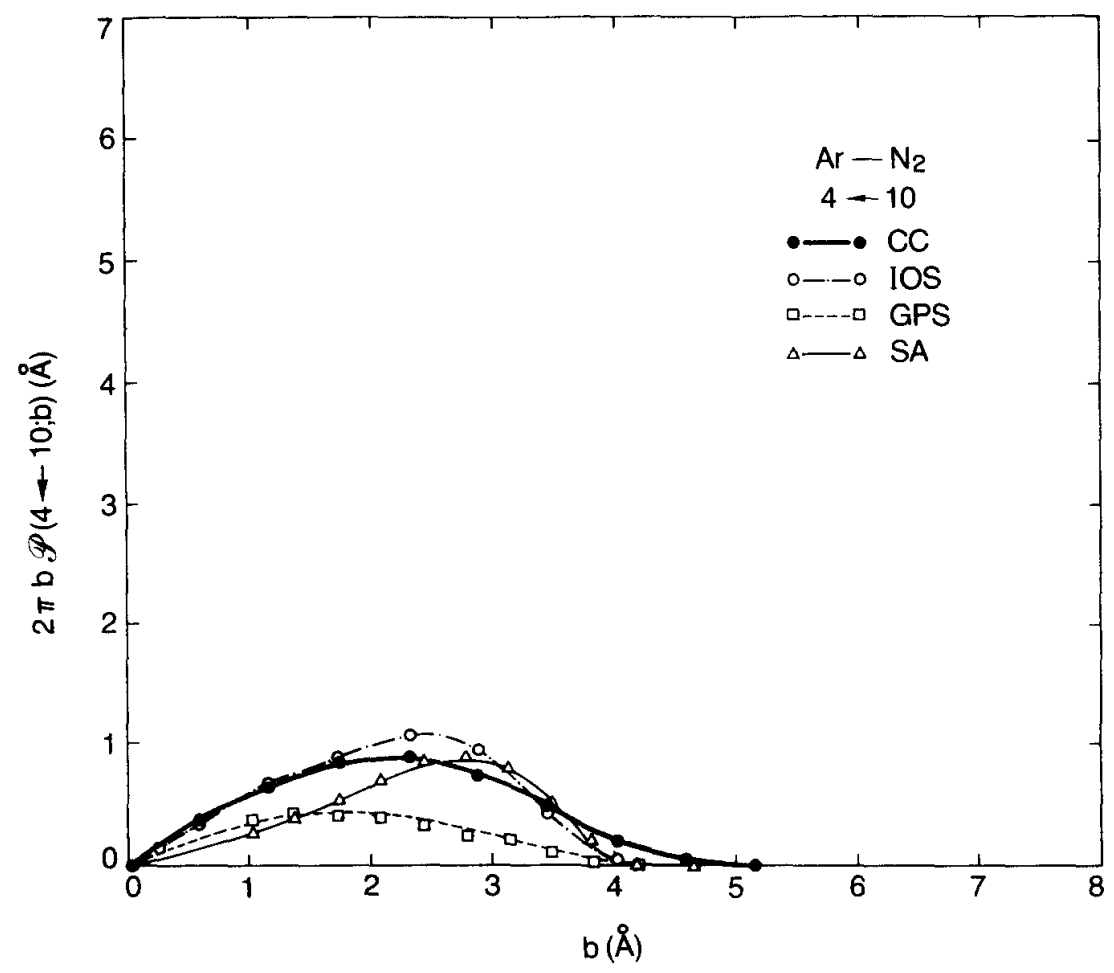

FIG. 1. Contributions $2 \pi b \odot(b)$ plotted vs impact parameter $b$ for the transition $4 \leftarrow 10$ in $\mathrm{Ar}-\mathrm{N}_{2}$ collisions at $E_{10} / k_{B}=450^{\circ} \mathrm{K}$. The area under the curves gives the integral cross section $\sigma(4-10)$. Heavy solid line and circles are $\mathrm{CC}$ results; dot-dash line and open circles is IOS; dashed line and squares is GPS; and light solid line and triangles is $\mathrm{SA}$. 
TABLE III. Accurate CC cross sections $\sigma\left(j^{\prime}-j\right)$ for $A r-N_{2}$ col lisions with $E_{10} / k_{B}=450^{\circ} \mathrm{K}$ and a different rotational basis set. The notation is that of Table I.

\begin{tabular}{ccccc}
\hline \hline$j^{\prime} j$ & 8 & 10 & 12 & 14 \\
\hline 8 & $\ldots$. & $(15.5)$ & $(4.7)$ & $(0.30)$ \\
10 & $(15.3)$ & 360 & 11.7 & $(1.26)$ \\
12 & $(3.9)$ & 9.8 & 425 & $(5.5)$ \\
14 & $(0.15)$ & $(0.62)$ & $(3.3)$ & $\ldots$ \\
$E_{j} / k_{B}\left({ }^{\circ} \mathrm{K}\right)$ & 560 & 450 & 317 & 161 \\
\hline
\end{tabular}

12,14 were included, so that 48 channels of one parity were involved and 44 channels of the other parity. These calculations were done at $J=0,10,20, \ldots 100$.

In each of these calculations, the tolerance parameters of the $\mathrm{CC}$ program were adjusted to make the $\mathrm{CC}$ transition probabilities (absolute squares of the elements of the S matrix) stable under change of the starting point of the numerical propagation, etc., to within about $1 \%$. The opacity functions (average transition probabilities) were obtained from the elements of the T matrix ( $\mathbf{T}=\mathbf{1}-\mathbf{S})$ using

$$
\odot_{J}\left(j^{\prime}-j\right)=(2 j+1)^{-1} \sum_{l=1}^{J+j} \sum_{J-j \mid}^{J+j^{\prime}} \sum_{l^{\prime}=\left|J-j^{\prime}\right|}\left|T^{J}\left(j^{\prime} l^{\prime} \mid j l\right)\right|^{2} \text {. }
$$

The integral cross sections are then given by

$$
\sigma\left(j^{\prime}-j\right)=\left(\pi / k_{j}^{2}\right) \sum_{J=0}(2 J+1) \mathscr{P}_{J}\left(j^{\prime}-j\right) .
$$

In performing this sum, a Simpson's rule numerical quadrature was used to interpolate between the $J$ values at which the $\mathrm{CC}$ calculations were done. The resulting cross sections agreed to within $2 \%$ in virtually all cases with those obtained using the trapezoidal rule instead. Thus, it is expected that most of the cross sections in Table I-III are accurate to within $3 \%$, and the number of significant figures reproduced in the tables is intended to represent that. However, from comparison of Tables II and III and from other test calculations that were done, it is clear that the cross sections involving "edge" values of $j(j=12$ in Tables I and II and $j=8$ and 14 in Table III) are not converged with respect to the rotational basis set. Also, the largest $J$ values at which calculations were performed was not large enough that the sum in (3) had converged for most of the elastic cross sections; some of these were estimated by extrapolation. And partial cross sections in a few cases involving low $j$ values oscillated sufficiently that the $\Delta J=10$ grid was too rough to get a good approximation to (3) with Simpson's rule. Accordingly, those cross sections in the tables that are thought uncertain by more than $4 \%$ but interesting for basis set comparisons or accurate enough to be useful for rough estimates are included in parentheses in the tables; the other uncertain ones are omitted.

In order to provide more detailed information for comparison with future calculations on this system than is obtainable from such highly averaged quantities as the integral cross sections, we present in Table IV the weighted opacity functions $(2 J+1) \odot_{J}\left(j^{\prime}-j\right)$ (for a few transitions) that were used in Eq. (3) in calculating the cross sections of Tables II and III. Opacity functions for the other transitions in those tables are available upon request from the author. It should be noted that although four figures are reproduced in Table IV, the numbers there are probably only accurate to about $1 \%$, and some of the very tiny numbers are probably accu-



J. Chem. Phys., Vol. 62, No. 8, 15 April 1975
FIG. 2. Contributions to $\sigma(6-10)$ vs impact parameter. The notation is that of Fig. 1. 
TABLE IV. Weighted opacity functions $(2 J+1) \Phi_{J}\left(j^{\prime}-j\right)$ from $\mathrm{CC}$ calculations for $\mathrm{Ar}-\mathrm{N}_{2}$ collisions at $E_{10} / k_{B}=450^{\circ} \mathrm{K}$. All are from the same calculation as Table II except the last column, which is from the same calculation as Table III. As explained in the text, not all the figures are significant.

\begin{tabular}{|c|c|c|c|c|c|c|c|c|}
\hline$J$ & $10 \div 0$ & $10 \div 2$ & $10-4$ & $10-6$ & $10-8$ & $10-10$ & $8-6$ & $12-10$ \\
\hline 0 & $0.1292 E-00$ & $0.2782 E-01$ & $0.2670 E-01$ & $0.1306 E-01$ & $0.2550 E-02$ & $0.5266 E-01$ & $0.6510 E-02$ & $0.2027 E-01$ \\
\hline 10 & $0.2567 E+01$ & $0.3400 E+01$ & $0.4517 E+01$ & $0.4988 E+01$ & $0.4874 E+01$ & $0.2734 E+02$ & $0.3728 E+01$ & $0.7404 E+01$ \\
\hline 20 & $0.4231 E+01$ & $0.5838 E+01$ & $0.8203 E+01$ & $0.9646 E+01$ & $0.9919 E+01$ & $0.5393 E+02$ & $0.7226 E+01$ & $0.1381 E+02$ \\
\hline 30 & $0.4664 E+01$ & $0.6905 E+01$ & $0.1063 E+02$ & $0.1377 E+02$ & $0.1567 E+02$ & $0.8221 E+02$ & $0.1101 E+02$ & $0.1846 E+02$ \\
\hline 40 & $0.3854 E+01$ & $0.6332 E+01$ & $0.1110 E+02$ & $0.1636 E+02$ & $0.2161 E+02$ & $0.1109 E+03$ & $0.1614 E+02$ & $0.1989 E+02$ \\
\hline 50 & $0.2352 E+01$ & $0.4491 E+01$ & $0.9386 E+01$ & $0.1620 E+02$ & $0.2565 E+02$ & $0.1683 E+03$ & $0.2341 E+02$ & $0.1751 E+02$ \\
\hline 60 & $0.9526 E+00$ & $0.2264 E+01$ & $0.6117 E+01$ & $0.1300 E+02$ & $0.2492 E+02$ & $0.1427 E+03$ & $0.3051 E+02$ & $0.1249 E+02$ \\
\hline 70 & $0.1901 E-00$ & $0.6328 E+00$ & $0.2522 E+01$ & $0.7530 E+01$ & $0.1923 E+02$ & $0.1812 E+03$ & $0.3183 E+02$ & $0.5081 E+01$ \\
\hline 80 & $0.7944 E-02$ & $0.4012 E-01$ & $0.3113 E-00$ & $0.1713 E+01$ & $0.8084 E+01$ & $0.2232 E+03$ & $0.2379 E+02$ & $0.2835 E-00$ \\
\hline 90 & $0.3839 E-05$ & $0.5001 E-04$ & $0.1388 E-02$ & $0.2409 E-01$ & $0.4473 E-00$ & $0.4026 E+03$ & $0.6609 E+01$ & $0.1783 E-03$ \\
\hline 100 & $0.5537 E-09$ & $0.2404 E-07$ & $0.1038 E-07$ & $0.2309 E-05$ & $0.2023 E-02$ & $0.5846 E+03$ & $0.1569 E-00$ & $0.5181 E-04$ \\
\hline 110 & $0.5539 E-08$ & $0.7657 E-09$ & $0.4652 E-09$ & $0.6196 E-08$ & $0.6891 E-04$ & $0.6352 E+03$ & $0.3687 E-03$ & \\
\hline 120 & & & & & $0.3935 E-04$ & $0.3913 E+03$ & $0.4861 E-03$ & \\
\hline 130 & & & & & $0.1641 E-04$ & $0.2088 E+03$ & $0.1543 E-03$ & \\
\hline 140 & & & & & $0.8968 E-05$ & $0.1088 E+03$ & $0.5065 E-04$ & \\
\hline 150 & & & & & & $0.5807 E+02$ & $0.1990 E-04$ & \\
\hline 160 & & & & & & $0.3172 E+02$ & & \\
\hline 170 & & & & & & $0.1776 E+02$ & & \\
\hline 180 & & & & & & $0.1031 E+02$ & & \\
\hline 190 & & & & & & $0.6199 E+01$ & & \\
\hline 200 & & & & & & $0.3854 E+01$ & & \\
\hline
\end{tabular}

TABLE V. IOS integral cross sections $\sigma\left(j^{\prime}-j\right.$ ) (in $\AA^{2}$ ) for $A r-N_{2}$ collisions with $E / k_{B}=300^{\circ} \mathrm{K}$. The rotational state basis included $j=0,2,4, \ldots, 22$. The last line gives the relative translational energy if the $\mathrm{N}_{2}$ is in state $j$ and emphasizes the degeneracy approximation.

\begin{tabular}{lccccccccc}
\hline \hline$j^{\prime} j$ & 0 & 2 & 4 & 6 & 8 & 10 & 12 & 14 & 16 \\
\hline 0 & 388 & 4.5 & 2.12 & 0.98 & 0.289 & 0.060 & 0.010 & 0.0013 & 0.0001 \\
2 & 22.3 & 400 & 11.3 & 5.2 & 2.10 & 0.58 & 0.118 & 0.019 & 0.0024 \\
4 & 19.1 & 20.4 & 399 & 11.7 & 5.6 & 2.36 & 0.68 & 0.140 & 0.0225 \\
6 & 12.7 & 13.5 & 16.8 & 398 & 11.8 & 5.9 & 2.55 & 0.74 & 0.156 \\
8 & 4.9 & 7.1 & 10.5 & 15.4 & 398 & 12.0 & 6.1 & 2.68 & 0.79 \\
10 & 1.26 & 2.45 & 5.5 & 9.5 & 14.8 & 397 & 12.1 & 6.2 & 2.77 \\
12 & 0.24 & 0.59 & 1.89 & 4.9 & 8.9 & 14.4 & 397 & 12.2 & 6.3 \\
14 & 0.037 & 0.108 & 0.45 & 1.66 & 4.6 & 8.6 & 14.1 & 396 & 12.2 \\
16 & 0.005 & 0.016 & 0.082 & 0.39 & 1.53 & 4.3 & 8.3 & 13.9 & 396 \\
$E_{y} / k_{B}\left({ }^{\circ} \mathrm{K}\right)$ & 300 & 300 & 300 & 300 & 300 & 300 & 300 & 300 & 300 \\
\hline \hline
\end{tabular}

TABLE VI. IOS integral cross sections for $E / k_{B}=450^{\circ} \mathrm{K}$. Other notation and conditions are the same as in Table V.

\begin{tabular}{cccccccccc}
\hline \hline$j^{\prime} j$ & 0 & 2 & 4 & 6 & 8 & 10 & 12 & 14 & 16 \\
\hline 0 & 334 & 3.5 & 1.64 & 0.95 & 0.36 & 0.093 & 0.018 & 0.0027 & 0.0003 \\
2 & 17.5 & 343 & 9.3 & 4.6 & 2.22 & 0.76 & 0.188 & 0.035 & 0.0053 \\
4 & 14.8 & 16.7 & 344 & 9.8 & 4.9 & 2.48 & 0.88 & 0.223 & 0.042 \\
6 & 12.4 & 11.9 & 14.1 & 342 & 9.8 & 5.1 & 2.66 & 0.97 & 0.247 \\
8 & 6.1 & 7.5 & 9.2 & 12.8 & 342 & 9.9 & 5.2 & 2.79 & 1.03 \\
10 & 1.96 & 3.2 & 5.8 & 8.2 & 12.2 & 342 & 9.9 & 5.3 & 2.88 \\
12 & 0.44 & 0.94 & 2.46 & 5.1 & 7.7 & 11.8 & 341 & 10.0 & 5.4 \\
14 & 0.078 & 0.204 & 0.72 & 2.2 & 4.8 & 7.4 & 11.6 & 341 & 10.1 \\
16 & 0.011 & 0.035 & 0.155 & 0.63 & 1.99 & 4.5 & 7.2 & 11.4 & 341 \\
$E_{j} / k_{B}\left({ }^{\circ} \mathrm{K}\right)$ & 450 & 450 & 450 & 450 & 450 & 450 & 450 & 450 & 450 \\
\hline \hline
\end{tabular}




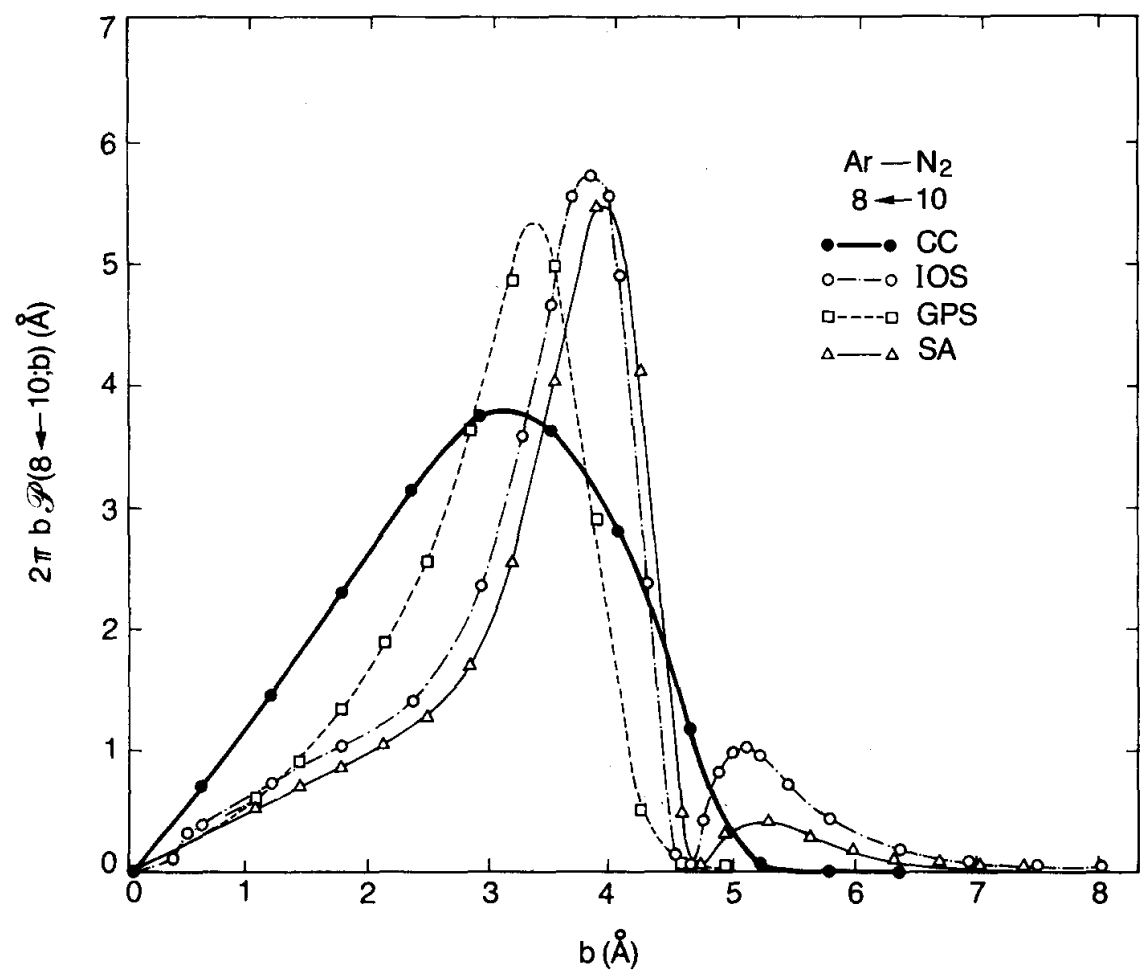

FIG. 3. Contributions to $\sigma(8 \leftarrow 10)$ vs impact parameter. The notation is that of

Fig. 1.

rate only to an order of magnitude at best.

Two sets of calculations in the IOS approximation are reported in Tables V and VI. (Several other IOS calculations were run to test basis set convergence, etc. and used to guide the way in which the $C C$ calculations were carried out.) In the two reported, the kinetic energies (in all the channels, since the rotational states are taken to be degenerate in this approximation) corresponded to 300 and $450^{\circ} \mathrm{K}$ for Tables $\mathrm{V}$ and VI, respectively. In both these cases, the calculation was carried out at the total angular momentum values, $J=0,2,4, \ldots, 200$ and (3) evaluated using the trapezoidal rule. In each, rotational states $j=0,2,4, \ldots 22$ were included. If spacefixed coordinates had been used, this would have required dealing with 144 channels of one parity and 132



FIG. 4. Contributions to $\sigma(12-10)$ vs impact parameter. The notation is that of

Fig. 1. 
TABLE VII. Comparison of accurate CC cross sections $\sigma(j-10)\left(\right.$ in $\left.\AA^{2}\right)$ for $\mathrm{Ar}-\mathrm{N}_{2}$ collisions with $E_{10} / k_{B}=450^{\circ} \mathrm{K}$ with the IOS approximation and with the results of Pattengill (preceding paper) for the classical trajectory (CT), generalized phase shift (GPS), sudden approximation (SA), and classical limit generalized phase shift (CGPS) methods.

\begin{tabular}{rccrrrr}
\hline \hline$j$ & CC & IOS & CT & GPS & SA & CGPS \\
\hline 0 & 0.094 & 0.093 & & & & \\
2 & 0.74 & 0.76 & 0.6 & & & 0.6 \\
4 & 2.35 & 2.48 & 2.2 & 0.9 & 1.8 & 0.8 \\
6 & 5.2 & 5.1 & 4.8 & 3.6 & 4.2 & 3.3 \\
8 & 10.9 & 9.9 & 13.2 & 8.6 & 8.3 & 11.7 \\
10 & 359 & 342 & & & & \\
12 & 9.8 & 11.8 & 9.6 & 8.6 & 10.2 & 9.8 \\
14 & $(0.6)$ & 7.4 & 1.0 & 4.4 & 6.1 & 4.8 \\
16 & 0.0 & 4.5 & 0.0 & 1.5 & 3.3 & 2.0 \\
\hline \hline
\end{tabular}

channels of the other parity and the diagonalization of $144 \times 144$ and $132 \times 132$ matrices at each $J$. However, in body-fixed coordinates ${ }^{4}$ it requires the one-time diagonalization of a total of only 23 matrices ranging in size from $1 \times 1$ to $12 \times 12$ at most. The only computational work of any consequence is the evaluation of 144 WKB phase shifts at each $J$, and we have previously shown how to do that very rapidly. ${ }^{4}$

It may be well to mention computer time requirements at this point. The 49-42 channel $\mathrm{CC}$ calculations of $\mathrm{Ta}-$ ble II required a total of $40 \mathrm{~min}$ of CDC 7600 time. The 144 - 132 channel IOS calculations of Table $\mathrm{V}$ required a total of $10 \mathrm{sec}$ of CDC 7600 time.

\section{DISCUSSION AND CONCLUSIONS}

In Table VII we compare the cross sections reported by Pattengill ${ }^{1}$ in the preceding paper with those obtained herein. The methods by which the cross section were calculated are, respectively, the accurate quantum mechanical close-coupling (CC) method, the infinite order sudden (IOS) approximation, exact classical trajectories (CT), generalized phase shift (GPS), full sudden approximation (SA), and classical limit generalized phase shift (CGPS) approximations.

Also, to provide a more detailed comparison, the $\mathrm{CC}$, IOS, GPS, and SA contributions to a few of the cross sections are plotted against impact parameter in Figs. 1-4. Although the quantum mechanical $\mathrm{CC}$ calculations do involve a number of different partial waves and hence different impact parameters at a single total angular momentum $J$, we can define an approximate impact parameter correct in an average sense as

$$
b=\left(J+\frac{1}{2}\right) / k_{j} .
$$

Then, letting $\odot_{J}\left(j^{\prime}-j\right)=\odot\left(j^{p}-j ; b\right)$ in (3), approximating the sum in (3) by an integral and changing to $b$ as the variable of integration, we obtain

$$
\sigma\left(j^{\prime}-j\right)=\int_{0}^{\infty} 2 \pi b \rho\left(j^{\prime}-j ; b\right) d b
$$

which gives $\sigma$ as the area under the curves in Figs. 1-4 and allows comparison with the semiclassical results of the preceding paper.

From Table VII and Figs. 1-4, it is seen that the IOS approximation, which is the simplest of the methods computationally, gives better cross sections for transitions to low $j$ than any of the other approximate methods. However, because of its assumption that the energy levels are degenerate, it gives increasingly worse results for transitions to higher $j$ and a rather large cross section for transitions into the energetically closed $j=16$ state.

Probably the most interesting observation from Table VII is that the classical trajectory results are better than any of the other approximate methods for transitions to higher $j$ and better than any except the IOS for transitions to lower $j$. They behave properly for both firstorder allowed $(\Delta j=2)$ and first-order forbidden transitions $(\Delta j>2)$, so that the method of assignment of trajectories to individual quantum states used in Ref. 5 is clearly working properly. Indeed, all the CT cross sections are fully accurate enough to be used for relaxation $^{6}$ calculations and virtually any other purpose.

Thus, we conclude that, for this system at least, the popular belief that the rotational motion of nonhydride diatomic molecules is essentially classical at room temperature is justified.

\section{ACKNOWLEDGMENTS}

The calculations reported herein were performed on the CDC 7600 computer at the Los Alamos Scientific Laboratory while the author was a visiting staff member there. The use of facilities, stimulating environment, and hospitality there are deeply appreciated. The patience and cooperation of Professor Merle Pattengill in getting this paper and the preceding one published together is also appreciated.

*Work performed in part under the auspices of the U. S. Atomic Energy Commission.

${ }^{1}$ M. D. Pattengill, J. Chem. Phys. 62, 0000 (1975), preceding paper.

${ }^{2}$ T. P. Tsien and R. T. Pack, Chem. Phys. Lett. 6, 54 (1970); 8, 579 (1971).

${ }^{3}$ T. P. Tsien, G. A. Parker, and R. T. Pack, J. Chem. Phys. 59, 5373 (1973).

${ }^{4}$ R. T. Pack, J. Chem. Phys. 60, 633 (1974).

${ }^{5}$ M. D. Pattengill, R. A. LaBudde, R. B. Bernstein, and C. F. Curtiss, J. Chem. Phys. 55, 5517 (1971).

${ }^{6}$ J. D. Russell, R. B. Bernstein, and C. F. Curtiss, J. Chem. Phys. 57, 3304 (1972).

${ }^{7}$ G. A. Parker, R. L. Snow, and R. T. Pack (to be published).

${ }^{8}$ R. G. Gordon, J. Chem. Phys. 51, 14 (1969); Program 187, Quantum Chemistry Program Exchange, Indiana University, 1969.

${ }^{9} \mathrm{~L}$. W. Hunter (preprint, December, 1974).

${ }^{10}$ D. Secrest, J. Chem. Phys, 62, 710 (1975).

${ }^{11}$ P. McGuire and D. J. Kouri, J. Chem. Phys. 60, 2488 (1974); P. McGuire, Chem. Phys. Lett. 23, 575 (1973).

${ }^{12}$ R. T. Pack, Chem. Phys. Lett. 14, 393 (1972). 